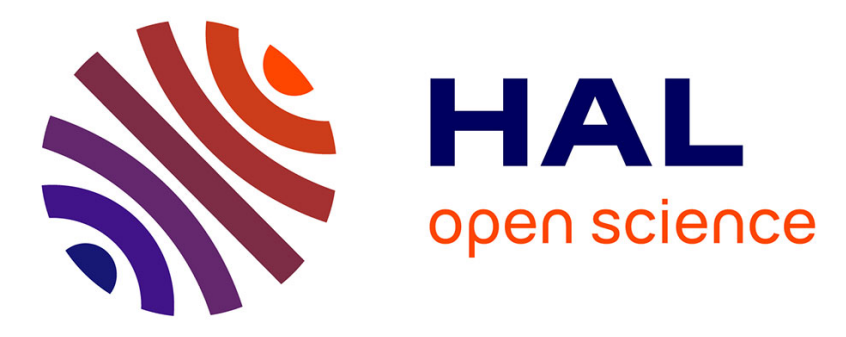

\title{
Pillars fabrication by DSA lithography: material and process options
}

Ahmed Gharbi, Patricia Pimenta-Barros, Olivia Saouaf, Gabriel Reynaud, Laurent Pain, Raluca Tiron, Christophe Navarro, Célia Nicolet, Ian Cayrefourcq, Michele Perego, et al.

\section{To cite this version:}

Ahmed Gharbi, Patricia Pimenta-Barros, Olivia Saouaf, Gabriel Reynaud, Laurent Pain, et al.. Pillars fabrication by DSA lithography: material and process options. Advances in Patterning Materials and Processes XXXV, Feb 2018, San Jose, United States. pp.105860Q, 10.1117/12.2297414 . cea02087039

\section{HAL Id: cea-02087039 \\ https://hal-cea.archives-ouvertes.fr/cea-02087039}

Submitted on 1 Apr 2019

HAL is a multi-disciplinary open access archive for the deposit and dissemination of scientific research documents, whether they are published or not. The documents may come from teaching and research institutions in France or abroad, or from public or private research centers.
L'archive ouverte pluridisciplinaire HAL, est destinée au dépôt et à la diffusion de documents scientifiques de niveau recherche, publiés ou non, émanant des établissements d'enseignement et de recherche français ou étrangers, des laboratoires publics ou privés. 


\title{
Pillars fabrication by DSA lithography: material and process options
}

\author{
A. Gharbi, P. Pimenta-Barros, O. Saouaf, G. Reynaud, L. Pain and R. Tiron \\ CEA-LETI, Minatec Campus, 17 rue des Martyrs, 38054 Grenoble cedex 9, France \\ C. Navarro, C. Nicolet and I. Cayrefourcq \\ ARKEMA France, Groupement de recherches de Lacq, RN 117, BP34- 64170 Lacq, France \\ M. Perego \\ MDM, IMM-CNR, Via C. Olivetti, 2, Agrate Brianza, Italy \\ F. Perez-Murano, E. Amat and M. Fernandez-Regulez \\ IMB, CSIC, 08193 Bellaterra, Spain
}

\begin{abstract}
We investigate the fabrication of sub-20 nm pillars by DSA lithography using PS-cylinder-forming PS- $b$-PMMA block copolymer (BCP). The approach is based on the removal of PMMA-matrix by either dry or wet etching to form PS pillars which act as a soft etching mask that can be further transferred to an intermediate hard one and then to the substrate. The process conditions of BCP self-assembly were optimized in terms of annealing temperature, brush layer composition and film thickness. It was demonstrated that PS/PMMA volume fractions of 50/50 in the PS-r-PMMA brush layer is the most adapted to obtain standing PS cylinders. Top-down SEM images showed a hexagonal array of PS cylinders in a PMMA matrix with a natural period of $33.5 \mathrm{~nm}$ (determined by Fast Fourier Transform FFT method) and $\mathrm{CD}$ around $15 \mathrm{~nm}$. Both wet and dry etching strategies for PMMA removal were discussed. It was shown that UV exposure followed by organic solvent development is necessary to decrease pattern collapsing during PMMA removal step which is the most critical issue for pillar fabrication. PMMA removal by dry etching was shown to completely avoid this pattern collapsing. Finally, pillars etching transfer to typical organic Si containing antireflective coating and spin on carbon (SiARC/SOC) hard mask and then to the silicon substrate was demonstrated. Si pillars of $15 \mathrm{~nm} \mathrm{CD}$ and $70 \mathrm{~nm}$ height were obtained with a straight profile shape.
\end{abstract}

Keywords: block copolymer, self-assembly, lithography, pillars

\section{INTRODUCTION}

Directed self-assembly (DSA) of block copolymers (BCPs) stands out as a promising and attractive patterning technique for nanoscale device fabrication in semiconductors and data storage applications [1-9]. It is still competing today with other emerging patterning approaches (Extreme UV lithography, nano-imprint lithography, parallel e-beam lithography) in order to replace or complement the conventional optical lithography in high volume manufacturing due to its easy processing, cost-effectiveness and fab-compatibility with current CMOS process flows [10-19]. Taking advantage of intrinsic chemical properties of BCPs, high-resolution, self-organized and well-oriented nano-domains with controlled dimensions $(10-50 \mathrm{~nm})$ and geometries (cylinders or lamellae) can be obtained after microphase separation leading to different types of features: contacts, pillars, lines and spaces. Over the last years, DSA has been extensively studied and many achievements have been demonstrated in patterning performances and devices fabrication. A main focus has been devoted to DSA process development using the industry standard Polystyrene-block-Poly (Methyl Methacrylate) (PS- $b$ PMMA) BCP due to its well-known properties and its compatibility of synthesis, processing and characterization with manufacturing. For line/space applications, the formation of $\sim 30 \mathrm{~nm}$-pitch fins by DSA using lamellar-morphology PS$b$-PMMA for FINFET transistor devices was demonstrated by several groups [1-4]. DSA line and space patterning

Advances in Patterning Materials and Processes XXXV, edited by Christoph K. Hohle, Proc. of SPIE Vol. 10586, 105860Q · @ 2018 SPIE · CCC code: 0277-786X/18/\$18 · doi: 10.1117/12.2297414 
performances were discussed in terms of critical dimension (CD) uniformity, pitch multiplication, defectivity and roughness using a high volume manufacturing (HVM) process flow. Silicon fins with $100 \mathrm{~nm}$ depth after transfer etching of PS mask with pre-determined customization were also demonstrated. Several other groups were interested in contact VIA patterning by DSA for logic or memories devices in sub-10 nm nodes [7-12]. Contact hole shrink and repair using cylindrical-morphology PS-b-PMMA BCP (PS as majority phase) have been shown with good CD uniformity, accurate alignment and good pattern fidelity after etching to hard mask sublayers. CD and pitch of $\sim 20 \mathrm{~nm}$ and $35-45 \mathrm{~nm}$ were reported, respectively. Concerning pillar fabrication by DSA, it was introduced as a potential HVM patterning technique for phase change material (PCM)-based memory devices [13, 14]. For that, cylindrical-morphology PS- $b$-PMMA BCP (PS as majority phase) was used to create $20-30 \mathrm{~nm}$ nanopores. After etching to dielectric hard mask, either atomic layer deposition (ALD) of PCM material or image reversal with metal and plasma etch transfer into a PCM film were performed to fabricate the PCM pillars. Similarly, sequential infiltration synthesis (SIS) of aluminum oxide $\left(\mathrm{Al}_{2} \mathrm{O}_{3}\right)$ in block copolymer has been also reported for pillars fabrication [20-22]. The approach is based on the selective infiltration of PMMA-cylinders with alumina followed by plasma dry etching of the PS-matrix. Hence, $\mathrm{Al}_{2} \mathrm{O}_{3}$ pillars are fabricated and used as a hard mask for transfer etching to adjacent layers. Although many achievements have been demonstrated in literature for contacts and lines patterning by DSA, process for pillar fabrication by DSA is still not so much investigated and was mainly based on the use of PMMA-cylinder forming PS- $b$-PMMA BCP and matrix inversion by the process.

In this work, we investigate the fabrication of sub-20 nm pillars by DSA lithography using PS-cylinder-forming PS- $b$ PMMA BCP. In this case, we are proposing to make the matrix inversion at the material level itself instead of doing it by the process and to push forward the approach capabilities in terms of etching and integration. We will focus on free surface patterning which allows us to make a fast evaluation of BCP materials and first tuning of the self-assembly process parameters. It is also a simple way to validate the masking robustness of realized pillars for plasma transfer etching.

\section{EXPERIMENTAL SET-UP}

\subsection{Materials}

Cylindrical-morphology Polystyrene- $b$-poly(methyl methacrylate) (PS- $b$-PMMA) di-block copolymer (BCP) with a PS/PMMA weight ratio of 30/70 was used in this paper (PMMA as a majority phase). The molecular weights of PS and PMMA are $17 \mathrm{Kg} / \mathrm{mol}$ and $45 \mathrm{~kg} / \mathrm{mol}$, respectively. Different PS- $r$-PMMA random copolymers with different PS and PMMA volume fractions were used as brush layers in order to control the BCP domains orientation.

The block and random copolymers are supplied by ARKEMA-BREWER SCIENCE under the commercial name OptiLign ${ }^{\mathrm{TM}}$.

\subsection{Process flow}

We are interested in this paper on BCP pillars fabrication on planar surface or what we also called 'free surface". First, the random copolymer PS- $r$-PMMA solution is spin coated on $300 \mathrm{~mm}$ wafer using RF3 track from Screen in order to obtain a film of about $50 \mathrm{~nm}$ thickness. The wafer is then annealed at $220^{\circ} \mathrm{C}$ for $2 \mathrm{~min}$ to ensure the chemical grafting of the material and rinsed in propylene glycol methyl ether acetate (PGMEA) during $1 \mathrm{~min}$ to remove the un-grafted random polymer chains. The resulting grafted random layer of around $10 \mathrm{~nm}$ thickness (also known as a brush neutral layer) is important to ensure the perpendicular orientation of PS cylinders to the substrate. Afterwards, the PS- $b$-PMMA diblock copolymer solution is spin-coated on the top of the random brush layer with different film thicknesses $(20 \mathrm{~nm}$, $35 \mathrm{~nm}$ and $50 \mathrm{~nm}$ ) and then baked to induce the self-assembly process. 


\subsection{Characterizations}

For the characterization, film thicknesses were measured with a Nanometrics ellipsometer. Top-view scanning electron microscopy (SEM) images after BCP self-assembly were taken by Hitachi H9300 or HCG4000 CD-SEMs with an accelerating voltage of $500 \mathrm{~V}$. Cross-sectional SEM images were obtained using a Hitachi S5000 SEM.

\section{RESULTS AND DISCUSSIONS}

For process optimization, we first investigated different PS/PMMA compositions of the brush layer (Figure 1). It was shown that PS/PMMA volume fractions of 50/50 in the brush layer is the most adapted to obtain PS- $b$-PMMA BCP selfassembly with a hexagonal array of standing PS cylinders within a PMMA matrix. The corresponding wetting angle contact of this brush layer is $76^{\circ}$. The result was validated at three different BCP film thicknesses. It has to be noted that the surface affinity of the underlayer was obviously shifted to higher PMMA affine for PMMA-cylinder forming BCP comparing to a PS-cylinder forming PS- $b$-PMMA BCP.

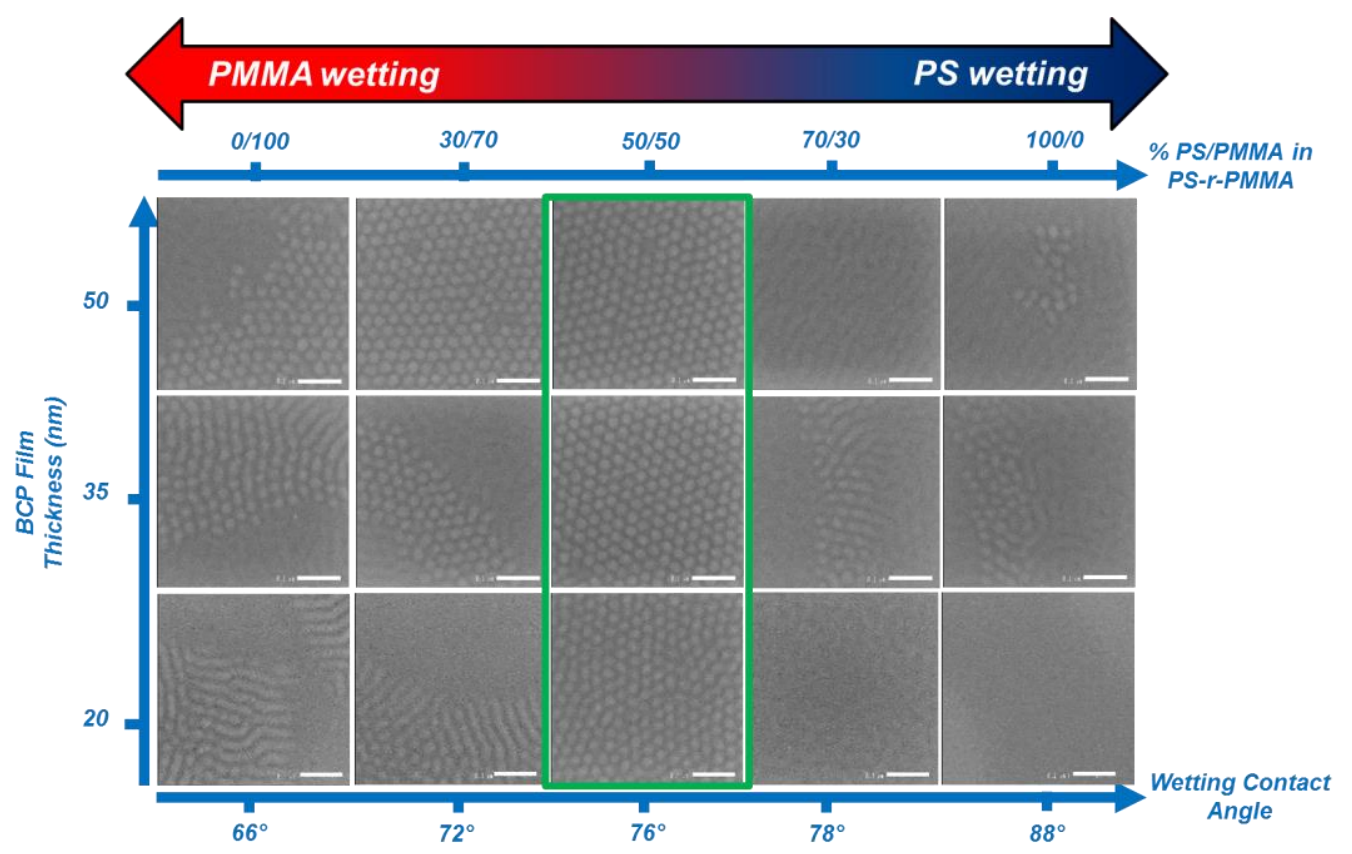

Figure 1. PS-r-PMMA brush underlayer optimization for PS cylinder forming PS- $b$-PMMA BCP. The best annealing conditions were chosen for each BCP thickness. The scale bar of SEM images is $100 \mathrm{~nm}$.

Then, we were interested in the optimization of annealing conditions of BCP self-assembly (bake time and temperature) at different thicknesses of 20,35 and $50 \mathrm{~nm}$, respectively (Figure 2). Thicker BCP film thickness is preferable for subsequent transfer etching but it is shown that higher thermal budget will be needed to ensure the phase separation and the self-assembly of BCP. Thicker the BCP film is, higher the needed annealing temperature for self-assembly is. For very high annealing temperature, the polymer was degraded and defect formation was seen as it is the case for the $35 \mathrm{~nm}$ film thickness at $300^{\circ} \mathrm{C}$ annealing temperature. It has to be also highlighted that higher thermal budget was needed for the self-assembly of a PS-cylinder forming BCP comparing to the PMMA-cylinder forming BCP. This can be related to the higher glass transition temperature and thus lower chain mobility for the higher PMMA-content material. 


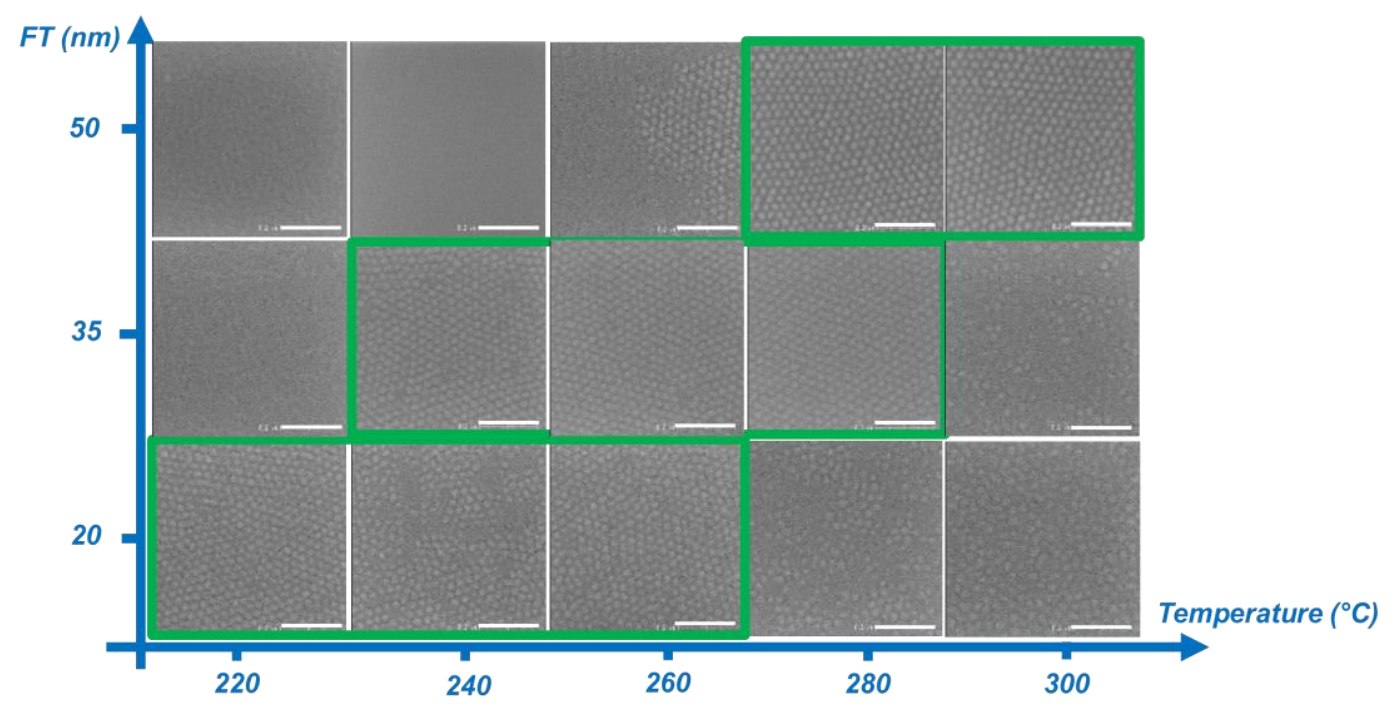

Figure 2. Process window (annealing temperature and BCP thickness) determination for $B C P$ selfassembly. A same annealing time of 5 min was used. Green boxes mean that good organization of perpendicular PS cylinders without obvious defects is obtained. The scale bar of SEM images is $200 \mathrm{~nm}$.

For an accurate determination of the self-assembly quality and kinetics, the ordering range length (or correlation length) was investigated through the measurement of grain-boundary-type defects using off-line treatment of SEM images with a home-made dedicated software. As shown in Figure 3, the software allows to distinguish between PS domains (in yellow in Figure 3b) and the PMMA matrix (in green in Figure 3b) after a first binarization step, leading to the localization of the coordinates of each centroid. Afterwards, the block copolymer natural period as well as pillars diameters distribution are determined. It has to be noted that the binarization step plays a significant role in the CD measurement. The threshold value which is, in fact, an essential parameter, must be constant throughout the entire set of analyses to guarantee a reliable comparison. Furthermore, the software enables the detection of grain boundaries and a "defectivity" measurement based on Delaunay triangulation analysis. Indeed, a pillar defect is characterized by having a number of nearest neighbors different than 6 (typically 5 or 7). In Figure 3c, the blue dots correspond to contacts considered to be good candidates. The red dots correspond to defects identified through the abnormal number of neighbors criteria provided by the Delaunay triangulation analysis [23].

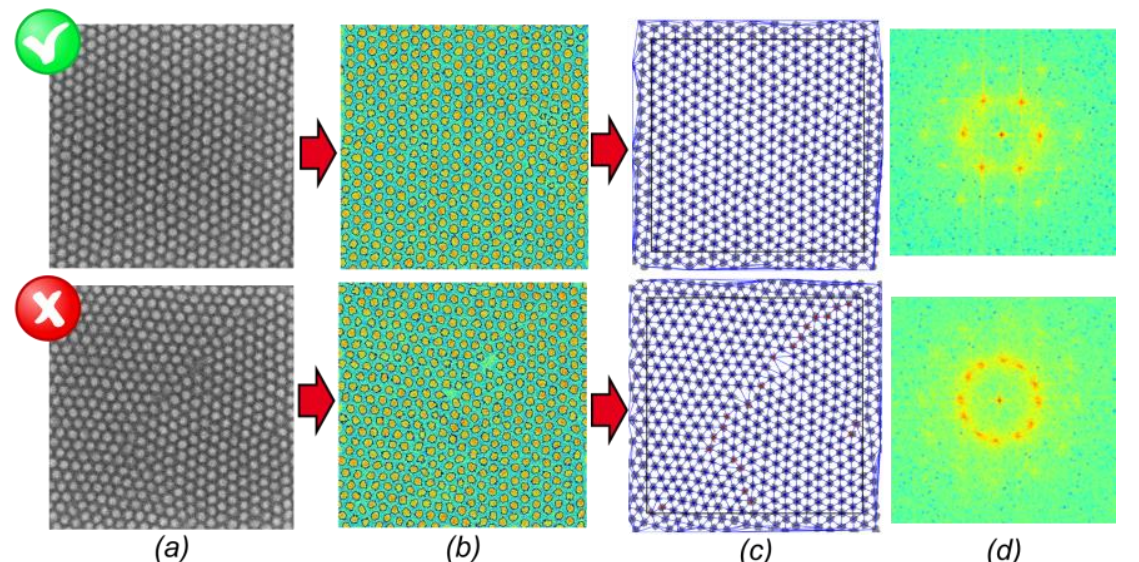

(a)

(b)

(c)

(d)

Figure 3. Image treatment and CD determination of self-assembled pillars: (a) raw image, (b) binarized and (c) CD measurement, (c) grain boundary defects detection and (d) Fast Fourier Transform FFT for natural period determination 
Figure 4 shows an example of grain number defects and natural period evolution with the annealing time for a BCP thickness of $50 \mathrm{~nm}$ and an annealing temperature of $280^{\circ} \mathrm{C}$. It is shown that a high level of defects is obtained for $1 \mathrm{~min}$ annealing with a morphology mixture of in-plane and out-of-plane cylinders. However, for higher annealing time (> 2 min), the self-assembly quality is enhanced and higher range ordering is observed. Surprisingly, the BCP natural period is showing a slight variation of around $3 \mathrm{~nm}$ with the annealing time. This behavior can be avoided by annealing under controlled atmospheric conditions using low oxygen hotplate as it has been reported by [24].

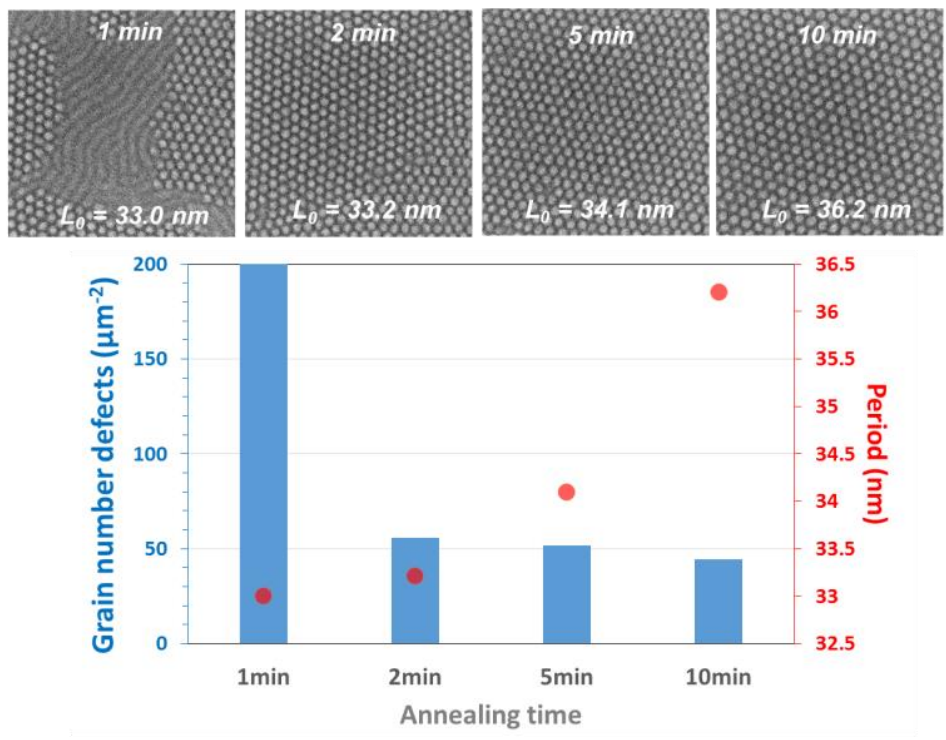

Figure 4. Grain number defects and natural period variation with annealing time. The annealing temperature and BCP film thickness are $280^{\circ} \mathrm{C}$ and 50 $n m$, respectively.

Once self-assembly conditions are optimized, the PMMA matrix must be selectively removed to perform PS pillars that can be transferred into under-layers by dry etching. In the literature, PMMA is known to be selectively removed with regards to PS in PMMA-cylinder-forming and lamellar-forming PS- $b$-PMMA BCP. Two different strategies have been reported. The first one is based on dry plasma etching using different chemistries such as oxygen, argon, or carbon monoxide [25-28]. The second technique used is wet development in acetic acid or adequate organic solvent after ultraviolet (UV) exposure [29-32]. In our case, both strategies are benchmarked for the PMMA removal in PS-cylinder forming PS- $b$-PMMA.

The main challenge of the wet etching is the collapsing of high aspect ratio patterns. By adjusting the UV exposure, complete and selective removal of PMMA by wet development in isopropanol (IPA) was achieved without high collapsing of PS pillars at less than $35 \mathrm{~nm} \mathrm{BCP}$ thickness (Figure 5). In fact, the UV radiation leads to the degradation of the PMMA-block via chains scission and the cleavage of the PS/PMMA covalent bonds. It also permits the cross-linking of PS and the reduction of its solubility. The wet development allows thus the selective removal of PMMA while preserving the thickness and shape of PS patterns. 


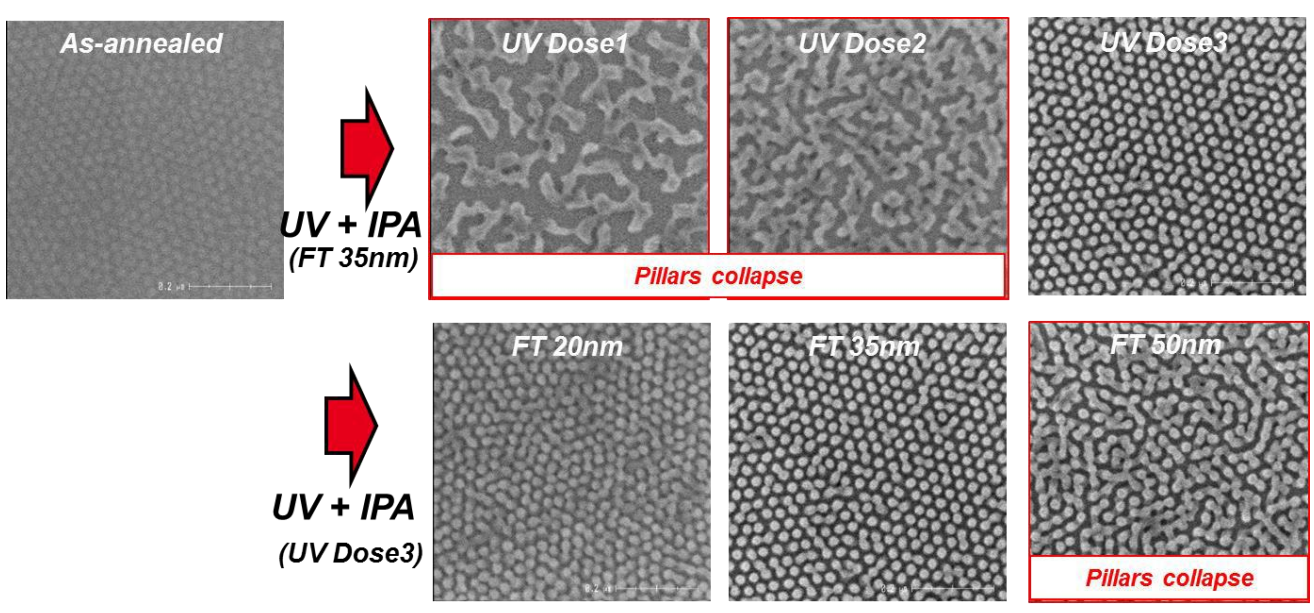

Figure 5. PMMA removal by UV exposure and isopropanol wet development for PS cylinder forming PS- $b$-PMMA BCP

On the other hand, we also used oxygen-based plasma dry etching for PMMA removal. The PS/PMMA dry etch selectivity was measured to be at 1.5 causing a PS thickness loss of around $20 \mathrm{~nm}$ after complete PMMA removal. Hence, higher BCP thickness was used $(50 \mathrm{~nm})$ leading to $\sim 30 \mathrm{~nm}$ height PS pillars which was enough for etching transfer to the SiARC/SOC layer and then to the substrate.

Figure 6 presents a comparison between dry etching and wet etching used for PMMA removal and subsequent transfer etching to Si through an intermediate SiARC/SOC hard mask layer. For both cases (wet and dry), PS pillars of $\sim 20 \mathrm{~nm}$ diameter were successfully transferred to up to $75 \mathrm{~nm}$ thickness of silicon. Moreover, it is clearly shown that dry PMMA removal allows better pattern fidelity after etching with no more collapsing of pillars comparing to wet removal.
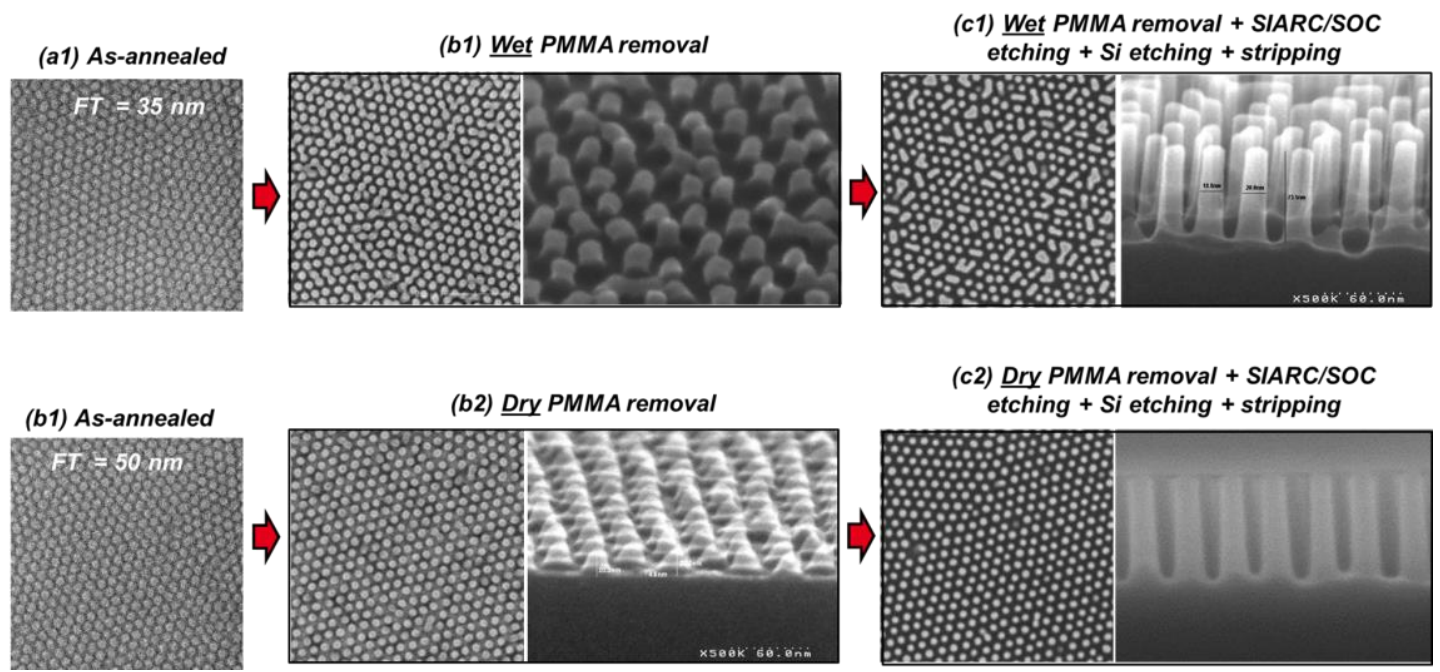

Figure 6. SEM and cross-sectional SEM of self-assembled pillars at different steps of the process: (a1, a2) as-assembled, (b1, b2) after PMMA removal (by dry or wet) and (c1, c2) after etching to Si and stripping using SiARC/SOC hard mask. 


\section{CONCLUSION}

We investigated pillars fabrication process using PS-cylinder-forming PS- $b$-PMMA BCP. The process conditions were optimized in terms of BCP self-assembly annealing temperature/time, brush layer composition and PMMA removal strategy. It was demonstrated that PS pillars were successfully fabricated after the selective removal of PMMA matrix by either UV-assisted wet development or plasma dry etching. The main challenge was the collapsing of PS pillars, particularly under wet etching. A maximum allowed BCP thickness for wet etching is $35 \mathrm{~nm}$ before patterns collapsing. For dry etching, no collapsing was observed after PMMA removal and higher initial thickness was needed for etching transfer to the substrate. Finally, PS pillars were successfully transferred to an intermediate SiARC/SOC hard mask and then to the Si substrate with a good pattern fidelity. High aspect ratio Si pillars were achieved: $15 \mathrm{~nm} C D, 75 \mathrm{~nm}$ height. In the future work, we will be interested in the fabrication of directed self-assembly (DSA) pillars using graphoepitaxy or chemoepitaxy approaches by taking into account the optimization performed in this work on free surface process.

\section{ACKNOWLEDGEMENTS}

The research leading to these results has received funding from the European Union's Horizon 2020 research and innovation program under grant agreement No 688072 IONS4SET. The authors also thank the CLG team, Brewer Science and Screen companies for their support.

\section{REFERENCES}

[1] H. Tsai et al. , ACS Nano, 8 (5), pp 5227-5232, 2014

[2] C. Liu et al., Proc. SPIE 9423, 94230S, 2015

[3] S. Sayan et al., Proc. SPIE 9779, 97790R, 2016

[4] S. Sayan et al., Proc. SPIE 9051, 90510M, 2014

[5] H.-C. Kim et al., Chem. Rev. 110(1), 146-177, 2009

[6] X.-Y. Bao et al., IEEE on Electron Devices Meeting (IEDM 2011), pp. 1-4, 2011

[7] W. Li and S. Yang, J. Vac. Sci. Technol. B 25(6), 1982-1984, 2007

[8] L.-W. Chang et al., Proc. SPIE 7637, 76370I, 2010

[9] H. Yi et al., Adv. Mat. 24, 3170-3114, 2012

[10] R. Tiron et al., Proc. SPIE 8680, 868012, 2013

[11] A. Gharbi et al., J. Micro/Nanolithography MEMS MOEMS 15(4), 043503, 2016

[12] L. Pain et al., Solid State Tech ᄀnol. 58(6), 14-18, 2015

[13] R-L. Bruce et al., Proc. SPIE 10149, 101490J, 2017

[14] C-C. Liu et al., Proc. SPIE 10146, 1014603, 2017

[15] M. Somervell et al., Proc. SPIE 9425, 94250Q, 2015

[16] C.-C. Liu et al., J. Vac. Sci. Technol. B 28(6), C6B30-C6B34, 2010

[17] J.Y. Cheng et al., ACS Nano 4(8), 4815-4823, 2010

[18] C. Bencher et al., Proc. SPIE 7970, 79700F, 2011

[19] L. Wan et al. ACS Nano, 9 (7), pp 7506-751, 2015

[20] J Frascaroli et al., ACS applied materials \& interfaces 8 (49), 2016

[21] Y.C Tseng et al., J. Phys Chem C, 115, 2011

[22] Peng et al., ACS Nano, 5 (6), 4600, 2011

[23] R. Tiron et al., JVST B, 29, 06F206, 2011

[24] M. Harumoto et al., Proc. SPIE 10146, 101461X, 2017

[25] P. Pimenta Barros et al., Proc. SPIE 9054, 90540G, 2014

[26] R.P. Kingsborough et al., Proc. SPIE 7271, 72712D, 2009

[27] C.C. Liu et al., J. Vac. Sci. Technol. B 25, 1963, 2007

[28] Y.H. Ting et al., J. Vac. Sci. Technol. B 26, 1684, 2008

[29] T. Thurn-Albrecht et al., Adv. Mater. 12, 787, 2000

[30] T. Xu et al., Adv. Funct. Mater. 13, 698, 2003

[31] M. Muramatsu et al., J. Micro-/Nanolithography, MEMS, MOEMS 11, 031305, 2012

[32] A. Gharbi et al., J. Vac. Sci. Technol. B, 33, 051602, 2015 